\title{
On the Role of Non-Gaussian Noises on Noise-Induced Phenomena
}

\author{
Horacio Wio
}

Most of the studies of noise-induced phenomena assume that the noise source is Gaussian (either white or colored). Here we present recent results of some of those noise-induced phenomena when driven by a noise source taken as colored and non-Gaussian, generated by a nonextensive $q$-distribution. In all the cases analyzed we have found that the response of the system is strongly affected by a departure of the noise source from the Gaussian behavior, showing an enhancement and/or a marked broadening of the corresponding system's response. The general result is that the value of the parameter $q$, optimizing the system's response, results in $q \neq 1$ (where $q=1$ corresponds to a Gaussian distribution). These results are of great relevance for many technological applications as well as for some situations of medical interest, like the noisy control of Wenckebach rhythms.

Nonextensive Entropy_Interdisciplinary Applications edited by Murray Gell-Mann and Constantino Tsallis, Oxford University Press 


\section{INTRODUCTION}

Fluctuations (or noise) have had a changing role in the history of science. We can identify three different stages. During the a first one, which lasted until the end of nineteenth century, noise was considered a nuisance to be avoided or eliminated. In the second stage, which started at the beginning of the twentieth century, it was possible to extract more information from a physical system through the study of fluctuations via Onsager, fluctuation-dissipation, and other related relations. The third stage corresponds to the last few decades of the twentieth century, with the recognition that in many situations noise can actually play a driving role that induces new phenomena. Some examples are noise-induced phase transitions [18, 27, 28], noise-induced transport [2, 29, 34, 35], stochastic resonance [17], and noise-sustained patterns [18].

Most of the studies on the noise-induced phenomena indicated above assume that the noise source is Gaussian (either white or colored). In addition to the intrinsic interest in the study of non-Gaussian noises, there is some experimental evidence, particularly in sensory and biological systems [3, 16, 20, 30, 32, 39], indicating that in at least some of these phenomena the noise sources could be non-Gaussian. The use of non-Gaussian noises in such studies is rare, mainly due to the difficulties of handling them and to the possibility of obtaining some analytical results when working with Gaussian (particularly white) noises.

Here we present recent results on some of those noise-induced phenomena when driven by a noise source taken as colored and non-Gaussian, generated by a Tsallis nonextensive $q$-distribution [10]. The phenomena we discuss here correspond to: stochastic resonance, gated trapping processes, Brownian motors, and, finally, the possibility of noisy control and elimination of Wenckebach rhythms [13].

In all the cases that were analyzed we have found that the response of the system is strongly affected by a departure of the noise source from the Gaussian behavior. For instance, in stochastic resonance we found an enhancement of the response (that is a larger maximum in the curve for the signal-to-noise ratio $[\mathrm{SNR}]$ ) and a marked broadening of the SNR curve indicating a larger degree of independence on the precise value of the noise intensity needed to tune the external signal. The general result is that the value of the parameter $q$ optimizing the system's response is $q \neq 1$ ( $q=1$ corresponding to a Gaussian distribution). These results have strong implications for technological and medical (cardiology) applications as well as for explaining some experimental and theoretical analyses on sensory systems.

In the next section we briefly discuss the form and properties of the nonGaussian noise source. After that we present the results for the different noiseinduced phenomena we have analyzed. In the last section we draw some conclusions. 


\section{NON-GAUSSIAN NOISE AND ITS PROPERTIES}

We start considering the following general form of a Langevin equation

$$
\dot{x}=-\frac{\partial U}{\partial x}+\eta(t) .
$$

However, at variance with other studies, we assume that the noise term $\eta(t)$ has a non-Gaussian distribution. Although we believe that our results are quite general, for concreteness and motivated by the work in Borland [4] based on a nonextensive thermostatistics distribution [10], we consider that the noise term is a Markovian process generated as the solution of the following Langevin equation

$$
\dot{\eta}=-\frac{1}{\tau} \frac{d}{d \eta} V_{q}(\eta)+\frac{1}{\tau} \xi(t),
$$

where $\xi(t)$ is a standard Gaussian white noise of zero mean and correlation $\left\langle\xi(t) \xi\left(t^{\prime}\right)\right\rangle$

$=D \delta\left(t-t^{\prime}\right)$, and

$$
V_{q}(\eta)=\frac{D}{\tau(q-1)} \ln \left[1+\frac{\tau}{D}(q-1) \frac{\eta^{2}}{2}\right] .
$$

The stationary properties of the noise $\eta$, including the time correlation function, have been studied in Fuestes et al. [15]. The stationary probability distribution is given by

$$
P_{q}^{s t}(\eta)=\frac{1}{Z_{q}}\left[1+\frac{\tau}{D}(q-1) \eta^{2}\right]^{\frac{-1}{q-1}}
$$

where $Z_{q}$ is the normalization factor. This distribution can be normalized only for $q<3$. The first moment, $\langle\eta\rangle=0$, is always equal to zero, and the second moment, $\left\langle\eta^{2}\right\rangle=2 D / \tau(5-3 q) \equiv D_{q}$, is finite only for $q<5 / 3$. Clearly, when $q \rightarrow 1$ we recover the limit of $\eta$ being a Gaussian colored noise (Ornstein-Uhlenbeck or OU process). Furthermore, for $q<1$, the distribution has a cut off and it is only defined for $|\eta|<\sqrt{2 D / \tau(1-q)}$. Finally, the correlation time $\tau_{q}$ of the process $\eta$ diverges near $q=5 / 3$ and it can be approximated over the whole range of values of $q$ as $\tau_{q} \approx 2 \tau /(5-3 q)$.

In Fuentes et al. [15], the above-indicated stochastic processes were analyzed in detail. Also, an effective Markovian approximation via a path integral procedure was obtained, making it possible to get quasi-analytical results for the mean-first-passage time or transition rate. Such results and their dependence on the different parameters in the case of a double-well potential were compared with extensive numerical simulations with excellent agreement.

In order to have an idea of the form of the stationary pdf [NEED TO DEFINE pdf] for $\eta$, in figure 1 we show it for different values of $q$, with $\beta=\tau / D$. 


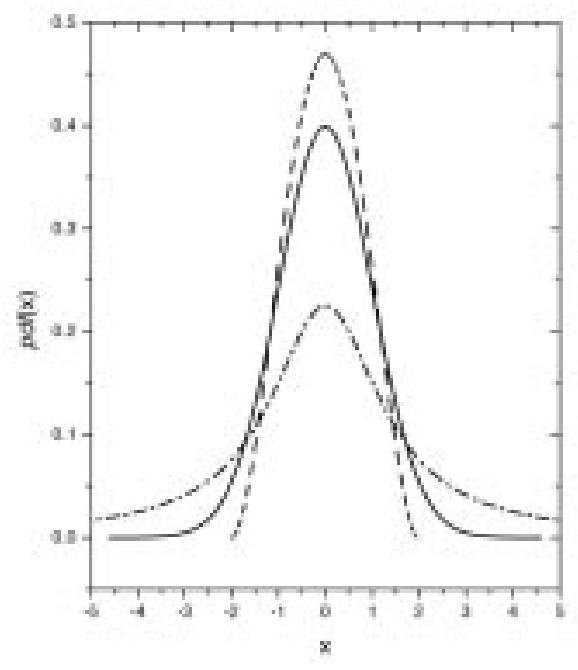

FIGURE 1 The stationary pdf given by eq. (4) with $\beta=1$. Solid line: Gaussian case $(q=1)$; dashed line: bounded distribution $(q=0.5)$; dashed-dotted line, wide distribution $(q=2)$.

\section{SOME APPLICATIONS}

\subsection{STOCHASTIC RESONANCE}

Stochastic resonance (SR) has attracted considerable interest due, among other aspects, to its potential technological applications for optimizing the output signal-to-noise ratio (SNR) in nonlinear dynamical systems, as well as to its connection with some biological mechanisms. The phenomenon shows the counterintuitive role played by noise in nonlinear systems as it enhances the response of a system subject to a weak external signal [17]. A tendency shown in recent papers, and determined by the possible technological applications, points toward achieving an enhancement of the system response (that is, obtaining a larger output SNR) by means of the coupling of several SR units in what forms an extended medium $[5,7,8,22,23,41]$.

A majority of studies on SR have been made analyzing a paradigmatic system: a bistable, one-dimensional, double-well potential. In almost all descriptions, and particularly within the two-state model (TST) [31], the transition rates between the two wells are estimated as the inverse of the mean first-passage time [31], which is evaluated using standard techniques, and most specifically through the Kramers approximation $[14,15]$. In all cases the noises are assumed to be Gaussian. 
Here we present the results of analyzing SR when the noise source is nonGaussian. We consider the following problem

$$
\begin{aligned}
\dot{x} & =f(x, t)+\eta(t), \\
\dot{\eta} & =-\frac{1}{\tau} \frac{d}{d \eta} V_{q}(\eta)+\frac{1}{\tau} \xi(t),
\end{aligned}
$$

where $\xi(t)$ is a Gaussian white noise as in eq. (2), and $V_{q}(\eta)$ is given as in eq. (3). The function $f(x, t)$ is derived from a potential $U(x, t)$, consisting of a double well potential and a linear term modulated by $S(t) \sim F \cos (\omega t)(f(x, t)=-d U / d x=$ $-U_{0}^{\prime}+S(t)$ ). This problem corresponds (for $\omega=0$ ) to the case of diffusion in a potential $U_{0}(x)$, induced by $\eta$, a colored non-Gaussian noise.

The details about the form of the effective Markovian Fokker-Planck equation can be found in Fuentes et al. $[14,15]$. There we calculated the stationary probability density, and derived the expression for the first passage time. We also exploited the TST approach [31] in order to obtain the power spectral density (psd) and the SNR.

In figure 2 we depict $R$ vs. $D$, for a fixed value of the time correlation $\tau$ $(\tau=0.1)$ and various $q$. The general trend is that the maximum of the SNR curve increases when $q<1$, this is when the system departs from the Gaussian behavior. Figure 3 again shows $R$ vs. $D$, but for a fixed value of $q(q=0.75)$ and several values of $\tau$. The general trend agrees with the results for colored Gaussian noises [17], where it was shown that the increase of the correlation time induces a decrease of the maximum of SNR as well as its shift toward larger values of the noise intensity. The latter fact is a consequence of the suppression of the switching rate with increasing $\tau$. Both qualitative trends are confirmed by Monte Carlo simulations of eq. (5). We have integrated eqs. (5) and (6) numerically using the Heun method. In all cases the results were obtained averaging over 2000 trajectories (5000 trajectories for $\tau=0$ ).

Figure 4 shows the simulation results for the same situation and parameters indicated in figure 2. Here, in addition to the increase of the maximum of the SNR curve for values of $q<1$, we see also an aspect that is not well reproduced or predicted by the effective Markovian approximation. It is the fact that the maximum of the SNR curve flattens for lower values of $q$, indicating that the system, when departing from Gaussian behavior, does not require a fine tuning of the noise intensity in order to maximize its response to a weak external signal. Figure 4 shows the simulation results for the same situation and parameters indicated in figure 2. Again, we found an agreement with the behavior found for colored Gaussian noises [17].

Our numerical and theoretical results indicate that:

1. for a fixed value of $\tau$, the maximum value of the SNR increases with decreasing $q$

2. for a given value of $q$, the optimal noise intensity (the one that maximizes SNR) decreases with $q$ and its value is approximately independent of $\tau$; and 


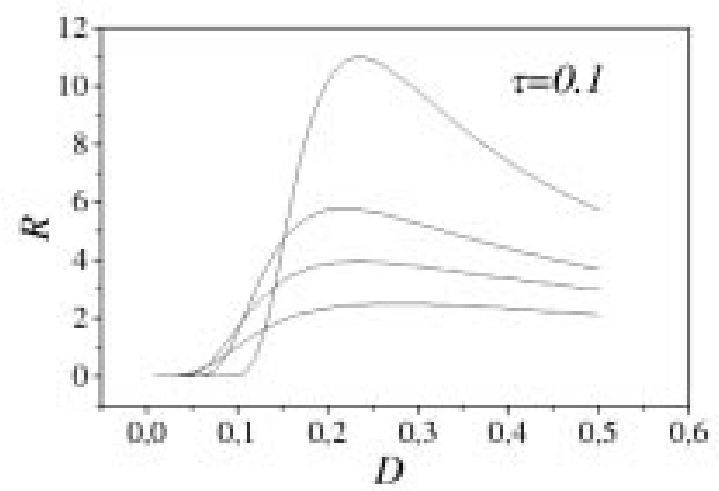

FIGURE 2 Theoretical value of SNR vs. $D$, for $\tau=0.1$ and the following values of $q=0.25,0.75,1.0,1.25$ (from top to bottom).

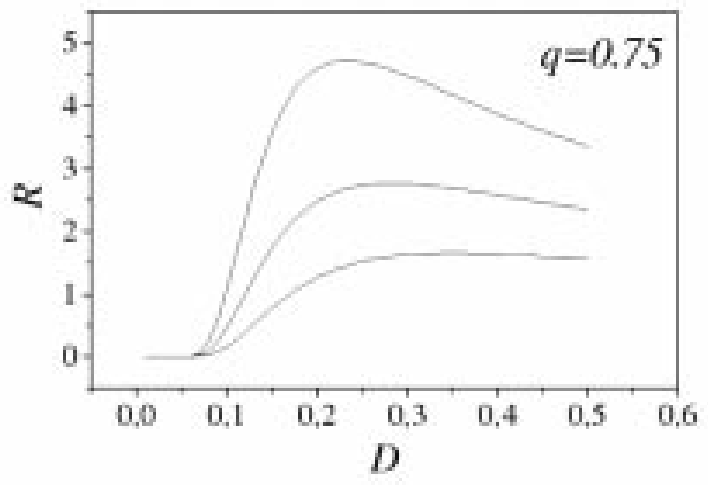

FIGURE 3 Theoretical value of SNR vs. $D$, for $q=0.75$ and the following values of $\tau=0.25,0.75,1.5$ (from top to bottom).

3. for a fixed value of the noise intensity, the optimal value of $q$ is independent of $\tau$ and, in general, it turns out that $q_{o p} \neq 1$.

In Castro et al. [9] we analyzed the case of SR when the noise source is nonGaussian, but from an experimental point of view. We studied an experimental setup similar to the one used in Fauve and Heslot [12], but we used a nonGaussian noise source that was built to exploit the form of noise introduced above, particularly white noise. Those results confirmed the predictions indicated above. 


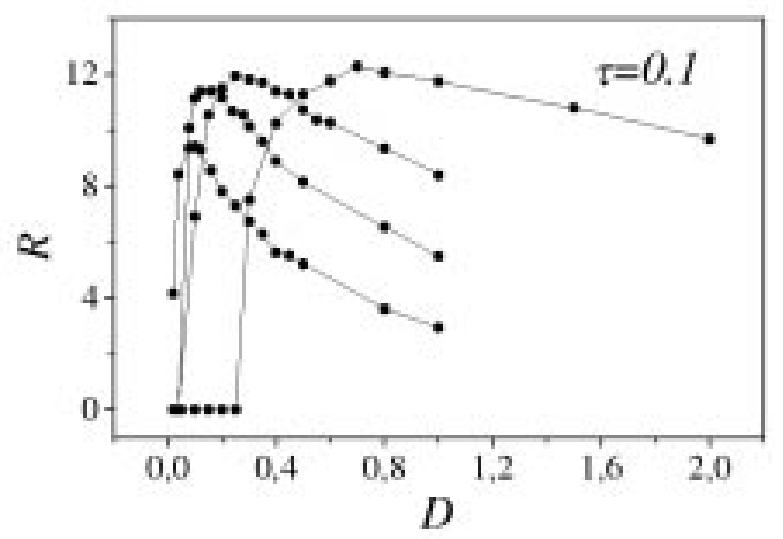

FIGURE 4 Simulation results of SNR vs. $D$, for $\tau=0.1$ and the following values of $q=0.25,0.75,1.0,1.25$ (from top to bottom).

\subsection{GATED TRAPS}

As indicated above, stochastic resonance has been found to play a relevant role in several problems in biology. In particular, there is an experiment related to the measurement of the current through voltage-sensitive ion channels in a cell membrane [3]. These channels switch (randomly) between open and closed states, thus controlling the ion current. This and other related phenomena have stimulated several theoretical studies of the problem of ionic transport through biomembranes, using different approaches, as well as different ways of characterizing stochastic resonance in such systems [16, 20,32].

In Sánchez et al. [37] we have studied a toy model, prompted by the work in Bezrukov and Vodyanoy [3], sketching the behavior of an ion channel. Among other factors, the ion transport depends on the membrane electric potential (which plays the role of the barrier height) and can be stimulated by both $d c$ and $a c$ external fields. This included the simultaneous action of a deterministic and a stochastic external field on the trapping rate of a gated imperfect trap. Rather than attempting a precise modeling of the behavior of an ionic channel, we proposed a simple model of dynamical trap behavior. Our main result was that even such a simple model of a gated trapping process shows SR-like behavior. In that initial study we assumed that the stochastic external field was a Gaussian white noise. Here, we sketch the main results, obtained analyzing the same model, but using a correlated non-Gaussian noise source, as detailed in Wio et al. [40].

In Sánchez et al. [37], the study was based on the so-called stochastic model for reactions $[1,36]$, generalized in order to include the internal dynamics of traps. The dynamical process consists of the opening or closing of the traps 


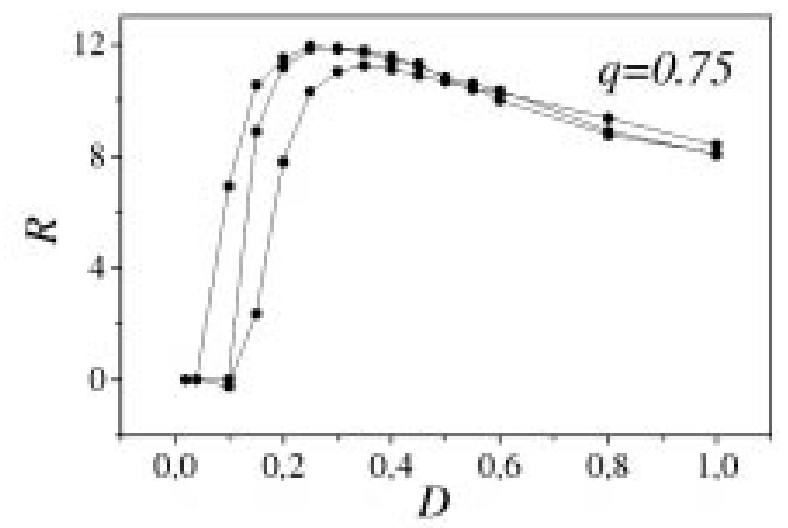

FIGURE 5 Simulation results of SNR vs. $D$, for $q=0.75$ and the following values of $\tau=0.25,0.75,1.5$ (from top to bottom).

according to an external field. Such a field has two contributions, one that is periodic with a small amplitude, and the other that is stochastic, the intensity of which will be (as usual) the tuning parameter. Here, to be consistent with the work in Sánchez et al. [37, 40], we change notation and use the same one as in those works. The starting model equation was

$$
\partial_{t} \rho(x, t)=D \partial_{x}^{2} \rho(x, t)-\gamma(t) \delta(x) \rho(x, t)+n_{u},
$$

where $\gamma$ is a stochastic process that represents the absorption probability of the trap, $\rho$ is the particle density (particles that have not yet been trapped). For a given realization of $\gamma$, here $D$ is the diffusion coefficient, $x$ is the coordinate over the one-dimensional system, and $n_{u}$ is a source term that represents a constant flux of ions. The injection of ions can be at a trap position or at any other position. In this last case the ion can diffuse to the trap position. This diffusion coefficient represents an effective diffusion through the volume rather than a diffusion over the membrane surface.

The absorption is modeled as $\gamma(t)=\gamma^{*} \theta\left[B \sin (\omega t)+\xi-\xi_{c}\right]$, where $\theta(x)$, the Heaviside function, determines when the trap is open or closed. The trap works as follows: if the signal, composed of the harmonic part plus $\xi$ (the noise contribution), reaches a threshold $\xi_{c}$, then the trap opens; otherwise, it is closed. We are interested in the case where $\xi_{c}>B$; that is, without noise the trap is always closed. When the trap is open the particles are trapped with a given frequency (probability per unit time) $\gamma^{*}$. In other words the open trap is represented by an "imperfect trap." Finally, in order to complete the model, we must give the statistical properties of the noise $\xi$. In Sánchez et al. [37] we assumed that $\xi$ is an uncor- 
related Gaussian noise of intensity $\xi_{0}$. In [40] we used a "colored" non-Gaussian noise given by the same eq. (6), where $\xi(t)$ is the non-Gaussian noise, $\xi_{w}$ is the white noise intensity and $V_{q}$ is given by $V_{q}(\xi)=1 / \beta(q-1) \ln \left[1+\beta(q-1) \xi^{2} / 2\right]$, where $\beta=\tau / \xi_{w}$.

We defined the current through the trap as $J(t)=\left\langle\gamma_{j}(t) \rho(j l, t)\right\rangle$. The brackets mean averages over all realizations of the noise. In Sánchez et al. [37], that is, in the case of $\xi(t)$ being a Gaussian white noise, we have obtained some analytical results and solved the equation numerically. However, for the non-Gaussian case [40] we should resort only to Monte Carlo simulations.

As in Sánchez et al. [37], we choose to quantify the SR-like phenomenon by computing the amplitude of the oscillating part of the absorption current given by $\Delta J=\left.J\right|_{\sin (\omega t)=1}-\left.J\right|_{\sin (\omega t)=-1}$. The qualitative behavior of the system can be explained as follows. For small noise intensities the current is low (remember that $\xi_{c}>B$ ); hence, $\Delta J$ is small, too. For a large noise intensity, the deterministic (harmonic) part of the signal becomes irrelevant and the $\Delta J$ is also small. Therefore, there must be a maximum at some intermediate value of the noise.

The details of the way the simulations were done can be found in Sánchez et al. [37] and Wio et al. [40]. All simulations shown in the figures correspond to averages over 1000 realizations. We have plotted all results as functions of the non-Gaussian noise intensity $\xi_{0}$. It is related to $\xi_{w}$ by $\xi_{0}=2 \xi_{w} /(5-3 q)$ [36].

In figure 6 we show the amplitude of the absorption current $\Delta J(t)$ as a function of the noise intensity $\xi_{0}$ for: (a) different values of $q$ and fixed $\tau$ and observational time $(t)$, and (b) for three different $\tau$ and fixed values of $q$ and $t$. The results are in agreement with those found in the case of Gaussian white noise. In the first case we see that the system response increases when $q<1$, and there is a shift of the maximum of $\Delta J(t)$ to larger noise values for increasing $q$. In the second case, the curves also show a shift of the maximum to larger noise intensities as $\tau$ increases. The shift of the $\Delta J(t)$ maximum with $\tau$ to larger values of $\xi_{0}$ is in agreement with a similar effect in "usual" SR [14, 15]. In that case, it was associated with the suppression of the switching rate with increasing $\tau$. In figure 7 we depict the maximum of $\Delta J(t)$ (which are averaged over the $\tau$ values for each $q$ ) for two different observational times as a function of the parameter $q$. For each observational time, the values are scaled with the corresponding maximum. We observe the existence of a new resonant-like maximum as a function of the parameter $q$. This implies that we can find a region of $q$ where the maximum $\Delta J$ reach optimal values (corresponding to a bounded and non-Gaussian pdf), yielding the largest system response.

The present results show that the use of non-Gaussian noises in the simple trapping process defined by eq. (7) produces significant changes in the system response when compared with the Gaussian case. In particular, we want to emphasize that we have found a double resonance-like phenomenon indicating that, in addition to an optimal noise intensity, there is an optimal $q$ value which yields the larger enhancement of the system response. The remarkable fact is that it corresponds to $q<1$ indicating that this enhancement occurs for a non-Gaussian 

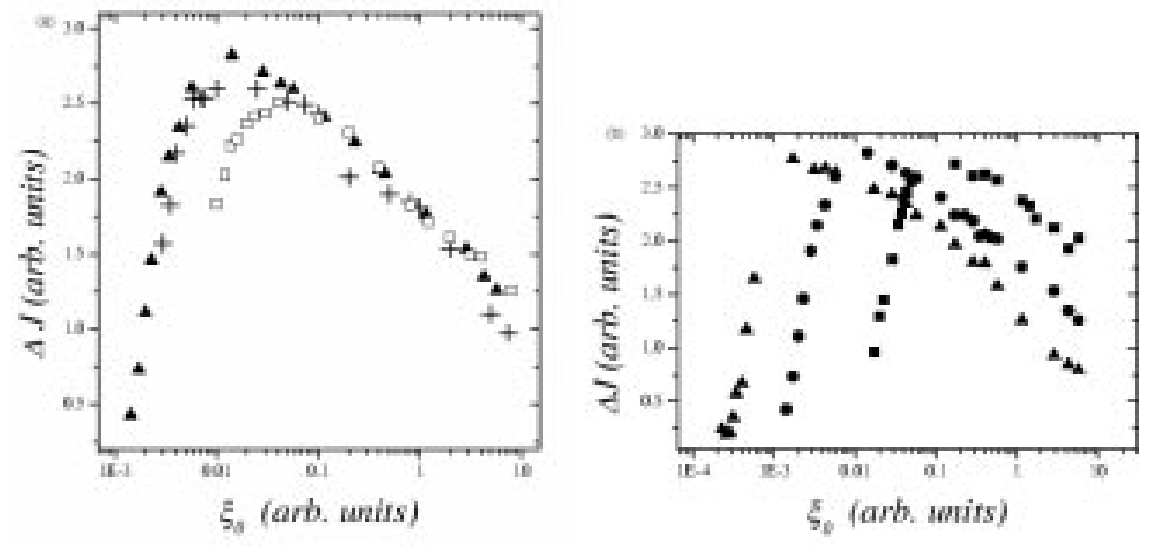

FIGURE 6 Value of $\Delta J$ (amplitude of the oscillating part of the absorption current) as a function of $\xi_{0}$ for a given observational time $(t=1140)$. (a) different values of $q$ (triangles $q=0.5$, crosses $q=1.0$, squares $q=1.5$ ) and a fixed value of $\tau(\tau=0.1)$. (b) different values of $\tau$ (triangles $\tau=0.01$, circles $\tau=0.1$, squares $\tau=1.0$ ) and a fixed value of $q(q=0.5)$.

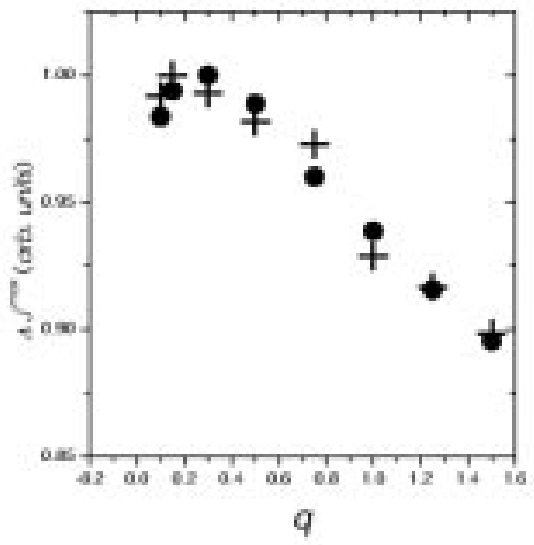

FIGURE 7 Dependence of $\Delta J_{\max }$, the value of $\Delta J$ at the maximum, as a function of $q$ and different observational times: circles $t=633$, crosses $t=1140$. 
and bounded distribution. The increase in the system response (here of $\Delta J(t)$ ) and the reduction in the need to tune the noise of $\xi_{0}$, can be understood as similar to the case of the usual SR [14, 15]. The bounded character of the pdf for $q<1$ contributes positively to the rate of overcoming the threshold $\xi_{c}$, and such a rate remains of the same order for a larger range of values of $\xi_{0}$.

\subsection{BROWNIAN MOTORS}

The study of noise-induced transport by "ratchets" has attracted the attention of an increasing number of researchers due to its biological interest and also to its potential technological applications [2, 34]. A recent new aspect has been to relax the requirement of a built-in bias: a system of periodically coupled nonlinear phase oscillators in a symmetric "pulsating" environment has been shown to undergo a noise-induced nonequilibrium phase transition, wherein the spontaneous symmetry breakdown of the stationary probability distribution function gives rise to an effective ratchetlike potential. Some of the striking consequences of this fact are the appearance of negative (absolute) zero-bias conductance in the disordered phase, but near the phase-transition line (for small values of the bias force $F$, the particle current $\langle\dot{X}\rangle$ opposes $F$ ), and anomalous hysteresis in the strong-coupling region of the ordered phase (the $\langle\dot{X}\rangle$ vs. $F$ cycle runs clockwise, as opposed, for instance, to the $B$ vs. $H$ cycle of a ferromagnet) $[29,35]$.

Here, in line with the work in Bouzat and Wio [6], we analyze the effect of the class of colored non-Gaussian noise introduced before on the transport properties of Brownian motors. We start considering the general system

$$
m \frac{d^{2} x}{d t^{2}}=-\gamma \frac{d x}{d t}-V^{\prime}(x)-F+\xi(t)+\eta(t),
$$

where $m$ is the mass of the particle, $\gamma$ the friction constant, $V(x)$ the ratchet potential, $F$ is a constant "load" force, and $\xi(t)$ the thermal noise satisfying $\left\langle\xi(t) \xi\left(t^{\prime}\right)\right\rangle=2 \gamma T \delta\left(t-t^{\prime}\right)$. Finally, $\eta(t)$ is the time-correlated forcing (with zero mean) that keeps the system out of thermal equilibrium by allowing rectification of the motion. For this type of ratchet model several different kinds of time-correlated forcing have been considered in the literature $[2,34]$. The main characteristic introduced by the non-Gaussian form of the forcing we consider here is the appearance of arbitrary strong "kicks" with relatively high probability when compared, for example, with the Gaussian OU process. As we shall see, in a general situation (without fine tuning of the parameters), this leads to the above-indicated enhancement effects.

We will consider the dynamics of $\eta(t)$ as described by the Langevin equation (2). As discussed before, for $1<q<3$, the probability distribution decays more slowly than a Gaussian, as a power law. Hence, keeping $D$ constant, the width or dispersion of the distribution increases with $q$, meaning that, the higher the $q$, the stronger the "kicks" that the particle will receive. 


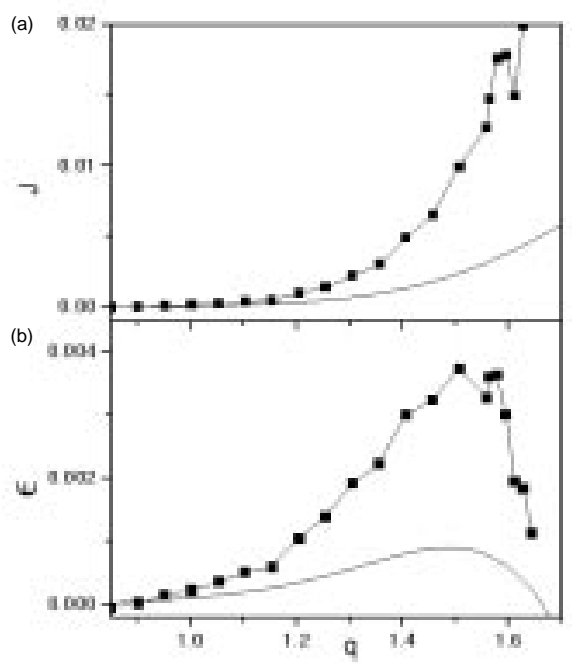

FIGURE 8 Current (a) and efficiency (b) as functions of $q$. The solid line corresponds to an adiabatic approximation, the line with squares shows results from simulations. All calculations are for $m=0, \gamma=1, T=0.5, F=0.1, D=1$, and $\tau=100 /(2 \pi)$.

For the ratchet potential we will first consider the same form as in Magnasco [26] (with period $2 \pi) V(x)=V_{1}(x)=-\int d x\left(\exp [\alpha \cos (x)] / J_{0}(i \alpha)-1\right)$, with $\alpha=16$. The integrand in $V(x)$ is the ratchet force $\left(-V^{\prime}(x)\right)$ appearing in eq. (8).

First, we have analyzed the overdamped regime setting $m=0$ and $\gamma=1$. Our intention was to analyze the dependence of the mean current $J=\langle d x / d t\rangle$ and the efficiency $\varepsilon$ on the different parameters, in particular, their dependence on the parameter $q$. As defined, $\varepsilon$ is the ratio of the work (per unit time) done by the particle "against" the load force $F\left(1 / T_{f} \int_{x=x(0)}^{x=x\left(T_{f}\right)} F d x(t)\right)$, to the mean power injected into the system through the external forcing $\eta\left(1 / T_{f} \int_{x=x(0)}^{x=x\left(T_{f}\right)} \eta(t) d x(t)\right)$. For the numerator we got $F\langle d x / d t\rangle=F J$, while, for the denominator, we obtained $1 / T_{f} \int_{0}^{T_{f}} \eta(t) d x / d t d t=1 / \gamma T_{f} \int_{0}^{T_{f}} \eta(t)^{2} d t=2 D\left[\gamma T_{f} \tau(5-3 q)\right]^{-1}$. Interesting and complete discussions on the thermodynamics and energetics of ratchet systems can be found in Parrondo et al. [33] and Sekimoto [38].

In figure 8 , we show typical analytical results for the current and the efficiency as functions of $q$, together with results coming from numerical simulations. Calculations have been done in a region of parameters similar to the one studied in Magnasco [26], but they consider (apart from the difference provided by the non-Gaussian noise) a non-zero load force that leads to a nonvanishing efficiency. As can be seen, although there is not a quantitative agreement between theory 
and simulations, the adiabatic approximation predicts qualitatively very well the behavior of $J$ (and $\varepsilon$ ) as $q$ is varied. As shown in the figure, the current grows monotonously with $q$ (at least for $q<5 / 3$ ), while there is an optimal value of $q(>1)$ which gives the maximum efficiency. This fact is interpreted as follows: when $q$ is increased, the width of the $P_{q}(\eta)$ distribution grows and high values of the non-Gaussian noise become more frequent, which leads to an improvement of the current. Although the mean value of $J$ increases monotonously with $q$, the grow of the width of $P_{q}(\eta)$ leads to an enhancement of the fluctuations around this mean value. This is the origin of the efficiency's decay that occurs for high values of $q$ : in this region, in spite of having a large (positive) mean value of the current, for a given realization of the process, the transport of the particle toward the desired direction is far from being assured. Hence, our results show that the transport mechanism becomes more efficient when the stochastic forcing has a non-Gaussian distribution with $q>1$.

Now we turn to the $m \neq 0$ case, that is, the situations in which the inertia effects are relevant. The results that we found [6] imply that separation of masses (particles with different masses moving in opposite directions) occurs, and that this happens in the absence of load force. In view of the results discussed above, it is reasonable to expect that non-Gaussian noises may improve the capability of mass separation in ratchets. Lindner et al. [24] was one of the primary works discussing mass separation by ratchets. There, the authors analyzed a ratchet system like the one described by eq. (8), considering OU noise as external forcing (in our case it corresponds to $q=1$ ). They studied (both numerically and analytically) the dynamics for different values of the correlation time of the forcing $\tau$, finding that there is a region of parameters where mass separation occurs. This means that the direction of the current is found to be mass dependent: the "heavy" species moves in the negative sense while the "light" one does so in the positive sense.

In order to compare results, we analyzed the same system studied in Lindner et al. [24, 25], but consider the non-Gaussian forcing. Hence, we studied eq. (8) with $V(x)=V_{2}(x)=-[\sin (2 \pi x)+0.25 \sin (4 \pi x)] /(2 \pi)$ as the ratchet potential. We focused on the region of parameters where, in Lindner et al. [24] (for $q=1$ ), separation of masses was found. We fixed $\gamma=2, T=0.1, \tau=0.75$, and $D=$ 0.1875 and considered the values of the masses $m=m_{1}=0.5$ and $m=m_{2}=1.5$ as in Lindner et al. [24]. Our main result was that the separation of masses is enhanced when a non-Gaussian noise with $q>1$ is considered. In figure 9(a) we show $J$ as function of $q$ for $m_{1}=0.5$ and $m_{2}=1.5$. It can be seen that there is an optimum value of $q$ that maximizes the difference of currents. This value, which is close to $q=1.25$, is indicated with a vertical double arrow. Another double arrow indicates the separation of masses occurring for $q=1$ (Gaussian OU forcing). We have observed that, when the value of the load force is varied, the difference between the curves remains approximately constant, but both are shifted together to positive or negative values (depending on the sign of the variation of the loading). By controlling this parameter it is possible to 


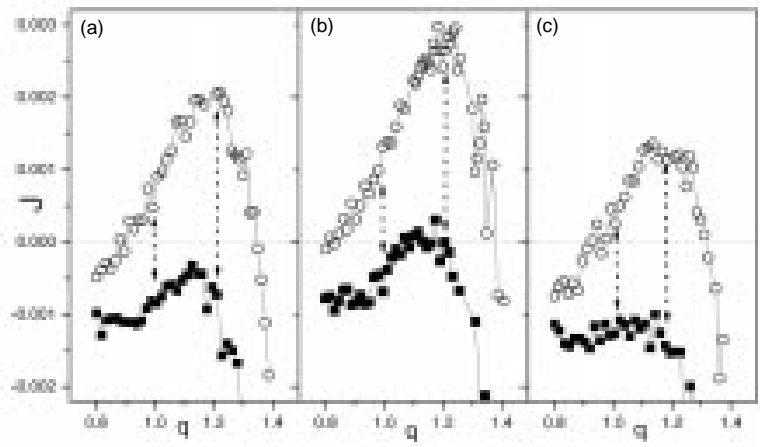

FIGURE 9 Separation of masses: results from simulations for the current as a function of $q$ for particles of masses $m=0.5$ (hollow circles) and $m=1.5$ (solid squares). Calculations for three different values of the load force: (a) $F=0.025$, (b) $F=0.02$, and (c) $F=0.03$.

achieve, for example, the situation shown in figure 9(b), where, for the value of $q$ at which the difference of currents is maximal, the heavy "species" remains static on average (has $J=0$ ), while the light one has $J>0$. Also, the situation shown in figure 9 (c), occurs when, for the optimal $q$, the two species move in the opposite direction with equal absolute velocity.

\section{FINAL REMARKS}

We have presented the results of a series of studies on noise-induced phenomena in which the noise source was non-Gaussian. In all cases, we have found that the system's response leads to enhancement of values of the parameter $q$, which indicates a departure from Gaussian behavior; that is, the optimum response happens for $q \neq 1$.

For the case of SR in a bistable system, we observed that the SNR, as we depart from Gaussian behavior (with $q<1$ ), shows two main aspects: first, its maximum as a function of the noise intensity increases; and second, it becomes less dependent on the precise value of the noise intensity. Both aspects are of great relevance for technological applications [17]. In addition to the increase in the response (SNR), the reduction in the need of tuning a precise value of the noise intensity is of particular relevance in order to understand how a biological system can exploit this phenomenon. The present results indicate that the noise model used here offers an adequate framework for analyzing such a problem. An analogous result occurs for the case of SR in the trap model. Finally, for the Brownian motor, what we have found is that a departure from Gaussian 
behavior, given now by a value of $q>1$, induces a remarkable increase in the current together with an enhancement of the motor efficiency. The latter shows, in addition, an optimum value for a given degree of non-Gaussianity. When inertia is taken into account, we also find a considerable increment in the mass separation capability.

Let us conclude by commenting on some related, although still preliminary, results. The problem that we want to address is associated with the so-called Wenckebach rhythms [19, 21]. In its classical manifestation, the Wenckebach phenomenon is characterized by a succession of electrocardiographic complexes in which atrioventricular (AV) conduction time (or P-R interval) increases progressively in decreasing increments until transmission failure occurs [19, 21]. In a recent contribution we analyzed the way Wenckebach rhythms arise in an excitable system due to the presence of defects (necrotic tissue) [13]. Afterward, we studied the possibility of eliminating or controlling the appearance of such rhythms through the injection of noise after or at the defect. Our intention was to determine the dependence of such rhythms on $q$, the parameter that controls the non-Gaussianity, and to analyze the optimum form of the noise to reach the control indicated above.

We used the simple FitzHugh model which involves only the membrane potential $u$ and a recovery variable $v$. The variable $v$ lumps together the timedependent activation of the potassium current with the time-dependent inactivation of the sodium current, in other words, the two slow variables of the Hodgkin-Huxley equation. In order to simulate the propagation of a wave train through the medium, induced by a periodic forcing, we have imposed a timedependent boundary condition for the variable $u$ at $x=0$, and have used initial conditions as discussed in Fuentes and Wio [13].

Even though our results are, so far, only preliminary and further studies are required, it is worth remarking here on what we have found: for a fixed (and low) noise intensity, the optimal form of noise for controlling Wenckebach rhythm production apepars to be results to be non-Gaussian (i.e., $q \neq 1$ ).

\section{ACKNOWLEDGMENTS}

I wish to thank S. Bouzat, F. Castro, M. Fuentes, M. Kuperman, J. Revelli, A. Sánchez, R. Toral, and C. Tsallis for their collaboration and/or useful discussions. Partial support from CONICET and ANPCyT, both Argentine agencies, is also acknowledged. I also want to thank Iberdrola S. A., Spain, for an award within the Iberdrola Visiting Professor Program in Science and Technology, and the IMEDEA and Universitat de les Illes Balears, Palma de Mallorca, Spain, for the kind hospitality extended to me. 


\section{REFERENCES}

[1] Abramson, G., A. Bru Espino, M. A. Rodriguez, and H. S. Wio. Phys. Rev. E. 50 (1994): 4319. [need title of paper for all references]

[2] Astumian, R. D. Science 276 (1997): 917.

[3] Bezrukov, S. M., and I. Vodyanoy. Nature 378 (1995): 362.

[4] Borland, L. Phys. Lett. A 245 (1998): 67.

[5] Bouzat, S., and H. S. Wio. Phys. Rev. E 59 (1999): 5142.

[6] Bouzat, S., and H. S. Wio. "Strong Enhancement of Current, Efficiency and Mass Separation in Brownian Motors Driven by Non-Gaussian Noises." Phys. Rev. Lett. submitted.

[7] Castelpoggi, F., and H. S. Wio. Europhys. Lett. 38 (1997): 91.

[8] Castelpoggi, F., and H. S. Wio. Phys. Rev. E 57 (1998): 5112.

[9] Castro, F. J., M. N. Kuperman, M. A. Fuentes, and H. S. Wio. Phys. Rev. E 64 (2001): 051105.

[10] Curado, E. M. F., and C. Tsallis. J. Phys. A 25 (1992): 1019.

[11] Dialynas, T. E., K. Lindenberg, and G. P. Tsironis. Phys. Rev. E 56 (1997): 3976.

[12] Fauve, S., and F. Heslot. Phys. Lett. A 97 (1983): 5.

[13] Fuentes, M. A., and H. S. Wio. Physica A 286 (2000): 345.

[14] Fuentes, M. A., R. Toral, and H.S. Wio. Physica A 295 (2001): 114-122.

[15] Fuentes, M. A., Horacio S. Wio, and Raúl Toral. Physica A 303 (2002): 91-104.

[16] Fuliński, A. Phys. Rev. Lett. 79 (1997): 4926.

[17] Gammaitoni, L., P. Hänggi, P. Jung, and F. Marchesoni. Rev. Mod. Phys. 70 (1998): 223.

[18] García-Ojalvo, J., and J. M. Sancho. Noise in Spatially Extended Systems. New York: Springer-Verlag, 1999.

[19] Glass, L., P. Hunter, and A. McCulloch, eds. Theory of the Heart. New York: Springer-Verlag, 1991.

[20] Goychuk, I., and P. Hänggi. Phys. Rev. E 61 (2000): 4272.

[21] Keener, J., and J. Sneyd. Mathematical Physiology. New York: SpringerVerlag, 1998.

[22] Lindner, J. F., B. K. Meadows, W. L. Ditto, M. E. Inchiosa, and A. Bulsara. Phys. Rev. Lett. 75 (1995): 3.

[23] Lindner, J. F., B. K. Meadows, W. L. Ditto, M. E. Inchiosa, and A. Bulsara. Phys. Rev. E 53 (1996): 2081.

[24] Lindner, R., L. Schimansky-Geier, P. Reimann, and P. Hänggi. In Applied Linear Dynamics and Stochastic Systems Near the Millenium, edited by J. B. Kadtke and A. Bulsara, 309. AIP, 1997.

[25] Lindner, R., L. Schimansky-Geier, P. Reimann, P. Hänggi, and M. Nagaoka. Phys. Rev. E 59 (1999): 1417.

[26] Magnasco, M. Phys. Rev. Lett. 71 (1993): 1477. 
[27] Mangioni, S., R. Deza, H. S. Wio, and R. Toral. Phys. Rev. Lett. 79 (1997): 2389.

[28] Mangioni, S. R. Deza, R. Toral, and H. S. Wio. Phys. Rev. E 61 (2000): 223.

[29] Mangioni, S., R. Deza, and H. S. Wio. Phys. Rev. E 63 (2001): 041115.

[30] Manwani, A. Ph.D. thesis, California Institute of Technology, Pasadena, CA, 2000.

[31] McNamara, B., and K. Wiesenfeld. Phys. Rev. A 39 (1989): 4854.

[32] Nozaki, D., D. J. Mar, P. Griegg, and J. D. Collins. Phys. Rev. Lett. 72 (1999): 2125.

[33] Parrondo, J. M. R., J. M. Blanco, F. J. Cao, and R. Brito. Europhys. Lett. 43 (1998): 248.

[34] Reimann, P. Phys. Rep. 361 (2002): 57.

[35] Reimann, P., R. Kawai, C. Van den Broeck, and P. Hänggi. Europhys. Lett. 45 (1999): 545.

[36] Sánchez, A. D., E. M. Nicola, and H. S. Wio. Phys. Rev. Lett. 78 (1997): 2244.

[37] Sánchez, A. D., J. A. Revelli, and H. S. Wio. Phys. Lett. A 277 (2000): 304.

[38] Sekimoto, K. Prog. Theor. Phys. Supl. 130 (1998): 17.

[39] Wiesenfeld, K., D. Pierson, E. Pantazelou, Ch. Dames, and F. Moss. Phys. Rev. Lett. 52 (1994): 2125.

[40] Wio, H. S., J. A. Revelli, and A.D. Sánchez. Physica D (2002): in press. [cond-mat/0109454].

[41] Wio, Horacio S., B. Von Haeften, and S. Bouzat. Special Issue: Proceedings of the Twenty-First IUPAP International Conference on Statistical Physics, STATPHYS21. Physica A 306C (2002): 140-156. 